\title{
GALAXY GROUPS: MORPHOLOGICAL SEGREGATION BETWEEN SPIRALS
}

\author{
G.Giuricin ${ }^{1}$, F.Mardirossian ${ }^{1}$, M.Mezzetti ${ }^{2}$, \\ A.Pisani ${ }^{3}$, M. Ramella ${ }^{2}$ \\ ${ }^{2}$ Dept. of Astronomy, Via Tiepolo 11, Trieste, Italy \\ ${ }^{2}$ Astronomical Observatory, Trieste, Italy \\ ${ }^{3}$ S.I.S.S.A., Strada costiera 11, Trieste, Italy
}

We have considered the sample of groups of galaxies
identified by M.J.Geller and J.P.Huchra (1983,
Astrophys.J.Suppl.52,61), omitting the groups which are part
of well-known clusters or those which are probably affected by the presence of interlopers. We have searched for possible morphological segregation among spirals in galaxy groups considering the mean-pairwise distance of the members of the galaxy system and the mean distance of the members from the center of the system. All the distances are normalized to the mean-pairwise distance (MPD) and to the mean distance from the center (CD), respectively, of all the members of the groups considered. We divided the family of the spirals (plus irregulars) into two subfamilies ( $S^{\prime}$ and $S^{\prime \prime}$ ); we included in $S^{\prime}$ the early-type spirals (with morphological type code $0 \leq T \leq 2$ ) and in $S^{n}$ the galaxies of the late morphological types (with $T \geq 3$ ). The median values of the ratios MPD(S')/MPD, MPD( $\left.S^{\prime \prime}\right) / M P D, C D\left(S^{\prime}\right) / C D, C D\left(S^{n}\right) / C D$ are $0.86,1.17,0.95,1.08$, respectively.

The distribution functions of the mean-pairwise distances MPD( $\left.S^{\prime}\right) / M P D$ and $M P D\left(S^{\prime \prime}\right) / M P D$, and of the mean distances from the center, $C D\left(S^{\circ}\right) / C D$ and $C D\left(S^{n}\right) / C D$, show the presence of segregation among $S^{\prime}$ and $S^{\prime \prime}$. According to the Kolmogorov-Smirnov statistics, the former couple of functions turns out to be significantly different at a confidence level of $\approx 96 \%$ and the latter at a level greater than 99\%. The mean-pairwise distance and the distance from the center of the group become larger and larger as one goes from $S$ ' to $S^{\prime \prime}$ galaxies.

Thus, morphological segregation among spirals appears to be shown not only in some superclusters and in some clusters, but also in a quite extended sample of galaxy systems characterized by relatively small sizes, as the groups of galaxies are. 